\title{
EVALUACIÓN DE LOS NIVELES \\ DE ILUMINACIÓN EN EMPRESAS \\ DE EXPLOTACIÓN DE ROCAS FOSFÓRICAS \\ PARA LA ELABORACIÓN DE PRODUCTOS \\ AGROINDUSTRIALES EN EL DEPARTAMENTO \\ DEL HUILA
}

\author{
Reinaldo Alfredo Victoria-Bonilla \\ https://orcid.org/0000-0001-9077-3477 \\ Corporación Universitaria Minuto de Dios, Colombia \\ NATALIA SANDOVAL-CASTRO \\ https://orcid.org/0000-0003-4035-6151 \\ Investigadora independiente, Colombia \\ RAMIRO GAMBOA-SUÁREZ \\ https://orcid.org/0000-0002-5727-6409 \\ Universidad Surcolombiana, Huila, Colombia
}

Recibido: 26 de mayo del 2020 / Aprobado: 28 de agosto del 2020

doi: https://doi.org/10.26439/ing.ind2020.n039.4914

RESUMEN: El presente estudio tiene como propósito evaluar los niveles de iluminación en las áreas de trabajo de empresas mineras dedicadas a la explotación de rocas fosfóricas para la elaboración de productos agroindustriales en el departamento del Huila. Para dicho estudio, se realizarán mediciones con un luxómetro, considerando la ubicación del terreno donde se encuentran las fábricas, la luz natural y la artificial. Estas mediciones se ejecutarán con el fin de evaluar las condiciones de iluminación que se encuentran en ese sector para determinar recomendaciones y así conseguir mejores resultados en las instalaciones, atendiendo a criterios de cantidad, calidad de luz y eficiencia energética. Posteriormente, en referencia a la normativa colombiana, estos resultados se analizarán, y con ello se determinará si las empresas cumplen con lo establecido para salvaguardar la seguridad y la salud de sus colaboradores.

Palabras clave: niveles de iluminación / áreas de trabajo / luxómetro / sector minero / productos agroindustriales / luxe

*Correos electrónicos: ravictoriab@gmail.com, sandovalcastronatalia@gmail.com, ramiro.gamboa@usco. edu.co 


\title{
EVALUATION OF LIGHTING LEVELS IN COMPANIES EXPLOITING PHOSPHORIC ROCKS FOR THE PRODUCTION OF AGRO-INDUSTRIAL GOODS IN THE DEPARTMENT OF HUILA
}

\begin{abstract}
The purpose of this study is to evaluate the lighting levels in the working areas of mining companies involved in the exploitation of phosphoric rocks for the production of agro-industrial goods in the department of Huila. To this end, measurements will be made with a luxometer, considering the location of the factories, and the natural and artificial lights. These measurements will be performed to evaluate the lighting conditions found in that sector, provide recommendations and thus achieve better results in the work stations according to light quantity and quality, and energy efficiency. Subsequently, these results will be analyzed based on the Colombian regulations to determine if the companies meet the rules that ensure the employees' safety and health.
\end{abstract}

Keywords: lighting levels / work areas / luxometer / mining sector / agro-industrial products / lux 


\section{INTRODUCCIÓN}

El departamento del Huila, ubicado al suroeste de Colombia, es considerado uno de los más privilegiados del país, pues su clima tropical permite el desarrollo de diferentes actividades económicas. Según la noticia "El Huila, una economía que diversifica” (2019), el presidente de la Cámara de Comercio, Ariel Rincón, manifiesta que Huila se ha convertido en un referente económico regional cada vez más diversificado. Según Molina y Ortiz (2019), gracias a la diversidad de procesos geológicos que dieron origen a las unidades litológicas presentes en el departamento de Huila, existe una alta variedad de depósitos minerales, recursos hídricos subterráneos y recursos energéticos que argumentan la definición del Huila como una zona estratégica de interés geológico, geomorfológico y ambiental para Colombia.

En el presente estudio, se evaluaron ocho de ellas: agrominerales, CINAGRO, Dolomita Rivera CIA y LTDA, Fosfatos del Huila, Minera Providencia, Minerales Reales del Sur y Montajes Técnicos de Colombia SAS, las cuales se dedican a la explotación y la transformación de rocas fosfóricas para la manufactura de productos agroindustriales.

La higiene industrial, según American Industrial Hygienist Association (1959), es la ciencia y arte dedicados al reconocimiento, evaluación y control de aquellos factores ambientales o tensiones emanados o provocados por el lugar de trabajo y que pueden ocasionar enfermedades, destruir la salud y el bienestar o crear algún malestar significativo entre los trabajadores o los ciudadanos de una comunidad. Asimismo, otros conceptos relevantes para el estudio son la seguridad y salud en el trabajo.

Según Melgar, Granados y Ruiz (2009), la seguridad en el trabajo es aquella situación en donde se reducen, en cuanto sea posible, los riesgos laborales mediante la correspondiente acción de prevención, y la salud en el trabajo es el estado de normatividad al que se aspira protegiendo al trabajador frente a posibles agenten insalubres o insano.

Se concluye que los conceptos mencionados previamente son disciplinas que se encargan de mitigar todas las condiciones laborales que pongan en peligro la salud y la seguridad de los empleados. Por eso, en el mundo existen organizaciones como la OIT (Organización Internacional del Trabajo) que se encarga y responsabiliza de la mejora permanente de las condiciones de trabajo. De la misma forma, en Colombia, se cuenta con una robusta normatividad legal que permite dar lineamientos constitucionales obligatorios para todas las organizaciones, ya sean públicas o privadas, como lo es implementar, y mantener el Sistema de Gestión de la Seguridad y Salud en el Trabajo. Así está consignado en el capítulo VI del Decreto $n .{ }^{\circ} 1072$ (2016) y se acompaña legislaciones como la Ley n. 9 (1979) y la Resolución 2400.

Además, teniendo en cuenta la industria en la que se desarrolla, esta investigación se rige por la Resolución n. ${ }^{\circ} 180466$ (2007), las guías técnicas colombianas de ICONTEC 
como la Guía Técnica Colombiana 8 (1995), la Guía Técnica Colombiana 45 (2012) y el Reglamento Técnico de iluminación y alumbrado RETILAP emitido en el año 2010, el cual indica que una adecuada iluminación es aquella que, además de satisfacer necesidades visuales y crear ambientes saludables, seguros y confortables, posibilita a los usuarios disfrutar de ambientes agradables, empleando los recursos tecnológicos más apropiados (Ministerio de Minas y Energía, 2010).

Cabe destacar que uno de los principales factores de riesgo presentes en este tipo de organizaciones es la iluminación, debido a que este factor se relaciona con la cantidad de luminosidad que se presenta en el sitio de trabajo y los estándares de iluminación se establecen según el tipo de tarea visual que los empleados deben ejecutar, teniendo en cuenta que, cuanto mayor sean los detalles y minucias, más necesaria será la luminosidad. En este sector de producción, las condiciones ambientales de los lugares o puestos de trabajo es uno de los factores más importantes, pues el 80 \% de la información se recibe por el campo visual. Es por ello que la iluminación puede impactar de forma positiva (buen ambiente laboral, mayor productividad) o una iluminación deficiente puede generar incomodidades, con los pertinentes perjuicios que esto representa para la salud de las personas, como serios problemas en los ojos, dentro de los cuales se encuentran la sequedad, picor, escozor, dolor de cabeza, cansancio, irritabilidad y mal humor, entre otros problemas (Chavarría, 2003, p. 6).

Es preciso realizar estudios que se encarguen de preservar las condiciones óptimas de trabajo, es decir, "aquellas que impiden que los trabajadores se vean afectados por factores laborales como las cargas físicas y mentales o los factores ambientales (biológicos, físicos, químicos, etc.) que pueden desarrollarse en el ámbito laboral", como expresan Ortega, Rodríguez y Hernández (2017).

Beltrán y Rodríguez (2019) indican que las actividades que se realizan con un nivel de iluminación inadecuado afectan directamente la productividad, la seguridad y el rendimiento, derivando en problemas de visión. Con la presente investigación se muestra cómo, mediante la aplicabilidad de las leyes, normas y otros factores, se realizan mediciones para evaluar las condiciones de iluminación de estas empresas del sector minero que se dedican a la fabricación de productos agroindustriales y, de esta manera, efectuar comparaciones.

\section{MATERIAL Y MÉTODOS}

Para el desarrollo de la presente investigación se definió una muestra constituida por las organizaciones agrominerales CINAGRO, Dolomita Rivera CIA y LTDA, Fosfatos del Huila, Minera Providencia, Minerales Reales del Sur, Montajes Técnicos de Colombia SAS. Para este constructo se hará un estudio de caso aplicado en cada empresa mencionada. 
El trabajo de campo se desarrolló en el año 2019 y tuvo una duración de seis meses, en los cuales se evaluaron áreas o lugares de trabajo en donde se realizan procesos o actividades como molienda, mezcla, secado, granulación, tolva, almacenamiento de producto terminado o laboratorio de control de calidad. Para el cumplimiento del objetivo general de esta investigación, que es realizar un estudio de iluminación, se propuso como metodología una investigación cuantitativa.

Para ello, se realizaron actividades para cumplir con los siguientes objetivos:

- Identificar y cuantificar los sitios y puestos de trabajo

- Cuantificar los niveles de iluminación general en cada uno de los puestos de trabajo

- Aplicar las normas establecidas según la normatividad legal

- Valorar las mediciones y compararlas con los valores recomendados por ICONTEC y la sociedad de ingenieros de iluminación IES

- Analizar y realizar recomendaciones a las empresas para mejorar la iluminación con el fin de disminuir los efectos por una deficiente o excesiva iluminación

Para el cumplimiento de los objetivos y actividades planteadas, en primer lugar se identificaron los puestos de trabajo, en los cuales se iban a realizar las mediciones, según la disponibilidad y los permisos de las empresas, y en los cuales se efectuaron las respectivas mediciones de intensidades de iluminación.

En la etapa de medición, se efectuó una inspección general a las instalaciones y puestos de trabajo, donde se evaluaron aspectos como la apariencia de las áreas físicas, las condiciones de los puntos de interés, la comodidad visual, el tipo de alumbrado, la limpieza del área y de las luminarias de alumbrado y la frecuencia como se realiza, el ambiente cromático, el deslumbramiento y las sombras. Por lo anterior, las mediciones desarrolladas en las áreas y puestos de trabajo de las industrias del estudio se basaron en los parámetros establecidos por el Ministerio de la Protección Social, en donde se enciende el luxómetro, revisando el estado de la batería y verificando que la pantalla indique cero con la fotocelda cubierta, seguidamente se selecciona la unidad de medición, la escala y el tipo de luz para continuar colocando la fotocelda sobre el plano del puesto de trabajo del operario, tomándose tres mediciones mínimo en el horario comprendido desde las 10:00 a las 14:00 horas. Luego, de los datos obtenidos en las mediciones se calculan el valor promedio y se compara con los valores recomendados para determinar que estén en el valor límite permisible que según la Resolución 2400 (1979), en su artículo 83 , se establecen los siguientes niveles: 
Tabla 1

Rangos de intervalos recomendados

\begin{tabular}{ll}
\hline Oficina & 300 a 500 lux \\
Bancos de trabajo & 500 a 1000 lux (diferenciación de detalles finos) \\
\hline
\end{tabular}

Elaboración propia

A partir de lo mencionado previamente, se realizó la medición, y para la interpretación de los resultados se tuvo en cuenta lo que se muestra en la tabla 2.

Tabla 2

Interpretación de la medición

\begin{tabular}{lll}
\hline N. ${ }^{\circ}$ & Calificación & Rango \\
\hline 1 & $\begin{array}{l}\text { Iluminación } \\
\text { adecuada }\end{array}$ & Niveles de iluminación dentro del rango permitido \\
& Iluminación & Niveles de iluminación por debajo del límite inferior del rango \\
deficiente & permitido \\
& Iluminación por & Niveles de iluminación por encima del límite superior del rango \\
& encima & permitido (solamente aplica para iluminación de tipo artificial) \\
& del intervalo & Si es de tipo natural no se considera perjudicial \\
\hline
\end{tabular}

Fuente: ICONTEC (1994)

Para las mediciones respectivas, se utilizó un equipo de medición de iluminación digital lux meter de marca Tondaj de referencia LX 1010B, con las especificaciones que se muestran en la tabla 3.

Tabla 3

Especificaciones generales del luxómetro

\begin{tabular}{ll}
\hline Pantalla & $18^{\prime \prime}(0,7 ")$ LCD \\
Rango & 0 a 50000 lux - 0 a 100000 lux \\
Tiempo de muestra & $0,4 \mathrm{~S}$ \\
Operación de temperatura & $0{ }^{\circ} \mathrm{Ca} 40{ }^{\circ} \mathrm{Co} 32^{\circ} \mathrm{F}$ a $104^{\circ} \mathrm{F}$ \\
Operación de humedad & 0 a $80 \% \mathrm{Rh}$ \\
Dimensión & $116 \times 70 \times 29 \mathrm{~mm}$ \\
Peso & $200 \mathrm{~g}$ \\
\hline
\end{tabular}

Fuente: The Calibration Centre (2016) 


\section{RESULTADOS}

Una vez conocidas las características de las áreas de trabajo tales como horma rectangular, localizadas sobre terreno plano y en un nivel, con pisos resistentes al choque y abrasión y que no sean resbaladizas, sus estructuras son de cubierta de techo en forma de armadura metálica con cubiertas en zinc, como también sus diseños, sin cerramientos y con una altura promedio de 10 a $12 \mathrm{~m}$ para atenuar la calor ambiental y mantener buena iluminación natural, sus lámparas son de tipo industrial de sodio, en un promedio de diez lámparas para las áreas de proceso y producto terminado distribuidas proporcionalmente y a una altura de las superficie que oscila entre 6 a $8 \mathrm{~m}$. Las actividades que se desarrollan en las industrias del sector para el área de materias primas e insumos son a cielo abierto, donde el material rajón o ripio se descarga por acción de volteo de las volquetas y que son movilizados por los operarios por medio de buguis o vagonetas al área de proceso donde por medio de molinos de martillos y paletas las piedras rajón se trituran hasta disminuir hasta diez micras, generando material particulado e impalpable, el cual se recoge por medio de extractores de campana y se almacenan en filtros de tela y silos, para pasar a unas zarandas que clasificaran el grano y finalizar con el almacenamiento en silos, dependiendo del tamaño del grano, para ser empacados en sacos de polipropileno de $50 \mathrm{~kg}$ para ser despachados en tractocamiones a los clientes.

Por todo lo anterior, las exigencias visuales y los requisitos del medio ambiente visual, que deben cumplir estas empresas del sector minero, están acordes a las necesidades y pasan por mediciones que habitualmente son objeto de medición para la evaluación y acondicionamiento ergonómico de la iluminación en puestos de trabajo, como la intensidad de lux. A continuación se presentan los resultados de dichos cálculos:

Tabla 4

Evaluación ambiental de iluminación agrominerales y abonos

\begin{tabular}{|c|c|c|c|c|c|c|c|c|c|}
\hline \multirow{2}{*}{$\begin{array}{l}\begin{array}{l}\text { Puesto de } \\
\text { trabajo }\end{array} \\
\text { Zona } \\
\text { industrial }\end{array}$} & \multicolumn{3}{|c|}{$\begin{array}{c}\text { Intensidad } \\
\text { de iluminación } \\
\text { (lux) }\end{array}$} & \multirow{2}{*}{$\begin{array}{c}\text { Nivel } \\
\text { prome- } \\
\text { dio (lux) } \\
842,33\end{array}$} & \multirow{2}{*}{$\begin{array}{c}\begin{array}{c}\text { Nivel reco- } \\
\text { mendado } \\
\text { (lux })\end{array} \\
750\end{array}$} & \multicolumn{3}{|c|}{$\begin{array}{l}\text { Criterio de uniformidad } \\
\frac{\text { Nivel promedio }}{\text { Nivel medio }}>0,66\end{array}$} & \multirow{2}{*}{$\begin{array}{c}\begin{array}{c}\text { Calificación } \\
\text { de la } \\
\text { iluminación }\end{array} \\
\text { Adecuada }\end{array}$} \\
\hline & 795 & 842 & 890 & & & 0,94 & 1,00 & 1,06 & \\
\hline
\end{tabular}

Elaboración propia

En la tabla 4 se observa que los resultados arrojados en relación con las medidas de intensidad de iluminación tomados se encuentran por encima del nivel recomendado de lux, pero según el criterio de uniformidad la calificación de la iluminación es adecuada, es decir, los niveles de iluminación están dentro del rango permitido. 
Tabla 5

Evaluación ambiental de iluminación Cinagro LTDA

\begin{tabular}{|c|c|c|c|c|c|c|c|c|c|c|c|}
\hline \multirow{2}{*}{\multicolumn{2}{|c|}{$\begin{array}{l}\begin{array}{l}\text { Puesto de } \\
\text { trabajo }\end{array} \\
\text { Tolva }\end{array}$}} & \multicolumn{3}{|c|}{$\begin{array}{c}\text { Intensidad } \\
\text { de iluminación } \\
\text { (lux) }\end{array}$} & \multirow{2}{*}{$\begin{array}{c}\text { Nivel } \\
\text { prome- } \\
\text { dio (lux) }\end{array}$} & \multirow{2}{*}{$\begin{array}{l}\begin{array}{c}\text { Nivel reco- } \\
\text { mendado } \\
\text { (lux) }\end{array} \\
750\end{array}$} & \multicolumn{4}{|c|}{$\begin{array}{l}\text { Criterio de uniformidad } \\
\frac{\text { Nivel promedio }}{\text { Nivel medio }}>0,66\end{array}$} & \multirow{2}{*}{$\begin{array}{c}\text { Calificación } \\
\text { de la } \\
\text { iluminación }\end{array}$} \\
\hline & & 6 & & 74 & & & 100 & 100 & & $s_{i}$ & \\
\hline \multirow{4}{*}{$\frac{\frac{c}{\varrho}}{\frac{\varrho}{0}}$} & Trituración & 930 & 20 & مח९९ & $890 \cap 0$ & 750 & 1,04 & 0,94 & 1, & $S_{i}^{\prime}$ & Adecuada \\
\hline & Molienda & 870 & 120 & 795 & 863,33 & 750 & T & 1,07 & 0, & Sí & Adecuada \\
\hline & Mezcla & 789 & 700 & 850 & 858,0 & 750 & 0,12 & 1,09 & 0, & Sí & Auecudud \\
\hline & Granulación & 910 & (050 & 980 & 906,67 & 750 & 1,00 & 0,92 & 1,08 & Sí & Adecuada \\
\hline
\end{tabular}

Elaboración propia

En la tabla 5 se observa que los resultados arrojados en relación con las medidas de intensidad de iluminación tomados se encuentran por encima del nivel recomendado de lux, pero según el criterio de uniformidad la calificación de la iluminación es ADECUADA, es decir, los niveles de iluminación están dentro del rango permitido.

Tabla 6

Evaluación ambiental de iluminación Dolomita del Huila

\begin{tabular}{|c|c|c|c|c|c|c|c|c|c|}
\hline \multirow{2}{*}{$\begin{array}{l}\text { Puesto de } \\
\text { trabajo } \\
\text { Tolva }\end{array}$} & \multicolumn{3}{|c|}{$\begin{array}{c}\text { Intensidad } \\
\text { de iluminación } \\
\text { (lux) }\end{array}$} & \multirow{2}{*}{$\begin{array}{c}\begin{array}{c}\text { Nivel } \\
\text { prome- } \\
\text { dio (lux) }\end{array} \\
869,33\end{array}$} & \multirow{2}{*}{$\begin{array}{c}\begin{array}{c}\text { Nivel reco- } \\
\text { mendado } \\
\text { (lux })\end{array} \\
750\end{array}$} & \multicolumn{3}{|c|}{$\begin{array}{l}\text { Criterio de uniformidad } \\
\frac{\text { Nivel promedio }}{\text { Nivel medio }}>0,66\end{array}$} & \multirow{2}{*}{$\begin{array}{c}\begin{array}{c}\text { Calificación } \\
\text { de la } \\
\text { iluminación }\end{array} \\
\text { Adecuada }\end{array}$} \\
\hline & 865 & 948 & 795 & & & 1,00 & 1,09 & $0,91 s$ & \\
\hline
\end{tabular}

Elaboración propia

En la tabla 6 se observa que los resultados arrojados en relación con las medidas de intensidad de iluminación tomados, se encuentran por encima del nivel recomendado de lux, pero según el criterio de uniformidad la calificación de la iluminación es ADECUADA, es decir, los niveles de iluminación están dentro del rango permitido. 
Tabla 7

Evaluación ambiental de iluminación fosfatos del Huila

\begin{tabular}{|c|c|c|c|c|c|c|c|c|c|c|}
\hline \multirow{2}{*}{$\begin{array}{c}\begin{array}{c}\text { Puesto de } \\
\text { trabajo }\end{array} \\
\text { Almacenamiento } \\
\text { producto } \\
\text { terminado }\end{array}$} & \multicolumn{3}{|c|}{$\begin{array}{c}\text { Intensidad } \\
\text { de iluminación } \\
\text { (lux) }\end{array}$} & \multirow{2}{*}{$\begin{array}{c}\text { Nivel } \\
\text { prome- } \\
\text { dio (lux) } \\
807,33\end{array}$} & \multirow{2}{*}{$\begin{array}{l}\text { Nivel reco- } \\
\text { mendado } \\
\text { (lux) }\end{array}$} & \multicolumn{4}{|c|}{$\begin{array}{l}\text { Criterio de uniformidad } \\
\frac{\text { Nivel promedio }}{\text { Nivel medio }}>0,66\end{array}$} & \multirow{2}{*}{$\begin{array}{l}\text { Calificación } \\
\text { de la } \\
\text { iluminación } \\
\text { Adecuada }\end{array}$} \\
\hline & 850 & 677 & 895 & & & 1,05 & 0,84 & 1,11 & Sí & \\
\hline Tolva & 651 & 891 & 765 & 769,00 & 750 & 0,85 & 1,16 & 0,99 & Sí & Adecuada \\
\hline Tolva Zaranda & 1100 & 950 & 1250 & 1100,00 & 750 & 1,00 & 0,86 & 1,14 & Sí & Adecuada \\
\hline $\begin{array}{l}\text { Clasificación ma- } \\
\text { terial estéril sobre } \\
\text { la banda }\end{array}$ & 1500 & 650 & 1105 & 1085,00 & 750 & 1,38 & 0,60 & 1,02 & Sí & Adecuada \\
\hline $\begin{array}{l}\text { Tolva producto } \\
\text { final }\end{array}$ & 1150 & 950 & 850 & 983,33 & 750 & 1,17 & 0,97 & 0,86 & Sí & Adecuada \\
\hline $\begin{array}{l}\text { Laboratorio de } \\
\text { Control de calidad }\end{array}$ & 1100 & 850 & 950 & 966,67 & 750 & 1,14 & 0,88 & 0,98 & Sí & Adecuada \\
\hline Retroexcavadora & 985 & 1217 & 1028 & 1076,67 & 750 & 0,91 & 1,13 & 0,95 & Sí & Adecuada \\
\hline
\end{tabular}

Elaboración propia

En la tabla 7 se observa que algunos los resultados arrojados en relación con las medidas de intensidad de iluminación tomados se encuentran por encima del nivel recomendado de lux, y en algunos casos por encima del límite permitido, pero teniendo en cuenta el criterio de uniformidad la calificación de la iluminación, el horario y las condiciones de iluminación en las que se encuentran estos lugares de trabajo la iluminación es ADECUADA, ya que para ser catalogada como ILUMINACIÓN POR ENCIMA DEL INTERVALO solo aplica para iluminación de tipo artificial, pues cuando es natural no se considera perjudicial.

Tabla 8

Evaluación ambiental de iluminación Minera Providencia

\begin{tabular}{|c|c|c|c|c|c|c|c|c|c|c|}
\hline \multicolumn{2}{|l|}{$\begin{array}{c}\text { Puesto de } \\
\text { trabajo }\end{array}$} & \multicolumn{2}{|c|}{$\begin{array}{c}\text { Intensidad } \\
\text { de iluminación } \\
\text { (lux) }\end{array}$} & \multirow{2}{*}{$\begin{array}{c}\text { Nivel } \\
\text { prome- } \\
\text { dio (lux) }\end{array}$} & \multirow{2}{*}{$\begin{array}{c}\begin{array}{c}\text { Nivel reco- } \\
\text { mendado } \\
\text { (lux) }\end{array} \\
750\end{array}$} & \multicolumn{4}{|c|}{$\begin{array}{l}\text { Criterio de uniformidad } \\
\frac{\text { Nivel promedio }}{\text { Nivel medio }}>0,66\end{array}$} & \multirow{2}{*}{$\begin{array}{c}\begin{array}{c}\text { Calificación } \\
\text { de la } \\
\text { iluminación }\end{array} \\
\text { Adecuada }\end{array}$} \\
\hline Molino & 1200 & 980 & 1150 & & & 1,08 & 0,88 & 1,04 & Sí & \\
\hline Mandíbula & & & & & & & & & & \\
\hline $\begin{array}{c}\text { Molino } \\
\text { martillos }\end{array}$ & 1050 & 1100 & 1220 & 1123,33 & 750 & 0,93 & 0,98 & 1,09 & Sí & Adecuada \\
\hline $\begin{array}{c}\text { Secado y } \\
\text { granulado }\end{array}$ & 1245 & 1070 & 1180 & 1165,00 & 750 & 1,07 & 0,92 & 1,01 & Sí & Adecuada \\
\hline
\end{tabular}

Elaboración propia 
En la tabla 8 se observa que los resultados arrojados en relación con las medidas de intensidad de iluminación tomados se encuentran por encima del nivel recomendado de lux, y en algunos casos por encima del límite permitido; sin embargo, considerando el criterio de uniformidad, la calificación de la iluminación, el horario y las condiciones de iluminación en las que se encuentran estos lugares de trabajo la iluminación es ADECUADA, ya que para ser catalogada como ILUMINACIÓN POR ENCIMA DEL INTERVALO solo aplica para iluminación de tipo artificial, pues cuando es natural no se considera perjudicial.

Tabla 9

Evaluación ambiental de iluminación Minerales Barios

\begin{tabular}{|c|c|c|c|c|c|c|c|c|c|c|c|}
\hline \multirow{2}{*}{\multicolumn{2}{|c|}{$\begin{array}{c}\text { Puesto } \\
\text { de trabajo } \\
\text { Molino }\end{array}$}} & \multicolumn{3}{|c|}{$\begin{array}{c}\text { Intensidad } \\
\text { de iluminación } \\
\text { (lux) }\end{array}$} & \multirow{2}{*}{$\begin{array}{c}\text { Nivel } \\
\text { prome- } \\
\text { dio (lux) } \\
848,33\end{array}$} & \multirow{2}{*}{$\begin{array}{l}\begin{array}{c}\text { Nivel reco- } \\
\text { mendado } \\
\text { (lux })\end{array} \\
750\end{array}$} & \multicolumn{4}{|c|}{$\begin{array}{l}\text { Criterio de uniformidad } \\
\frac{\text { Nivel promedio }}{\text { Nivel medio }}>0,66\end{array}$} & \multirow{2}{*}{$\begin{array}{c}\text { Calificación } \\
\text { de la } \\
\text { iluminación }\end{array}$} \\
\hline & & 795 & 840 & 910 & & & 0,94 & 0,99 & 1,07 & Sí & \\
\hline \multirow{3}{*}{ 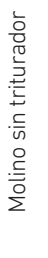 } & $\begin{array}{l}\text { Pesado de } \\
\text { bultos } 1\end{array}$ & 810 & 860 & 900 & 856,67 & 750 & 0,95 & 1,00 & 1,05 & Sí & Adecuada \\
\hline & $\begin{array}{l}\text { Pesado de } \\
\text { bultos } 2\end{array}$ & 945 & 850 & 790 & 861,67 & 750 & 1,10 & 0,99 & 0,92 & Sí & Adecuada \\
\hline & $\begin{array}{c}\text { Tolva } \\
\text { alimenticia }\end{array}$ & 830 & 920 & 875 & 875,00 & 750 & 0,95 & 1,05 & 1,00 & Sí & Adecuada \\
\hline
\end{tabular}

Elaboración propia

En la tabla 9 se observa que los resultados arrojados en relación con las medidas de intensidad de iluminación tomados se encuentran por encima del nivel recomendado de lux, pero según el criterio de uniformidad la calificación de la iluminación es ADECUADA, es decir, los niveles de iluminación están dentro del rango permitido.

Tabla 10

Evaluación ambiental de iluminación Minerales del Sur

\begin{tabular}{|c|c|c|c|c|c|c|c|c|c|c|}
\hline $\begin{array}{c}\text { Puesto } \\
\text { de trabajo } \\
\text { Molino 4: }\end{array}$ & & \multicolumn{2}{|c|}{$\begin{array}{c}\text { Intensidad } \\
\text { de iluminación } \\
\text { (lux) }\end{array}$} & $\begin{array}{l}\text { Nivel } \\
\text { prome- } \\
\text { dio (lux) }\end{array}$ & $\begin{array}{l}\text { Nivel reco- } \\
\text { mendado } \\
\quad(l u x)\end{array}$ & \multicolumn{4}{|c|}{$\begin{array}{l}\text { Criterio de uniformidad } \\
\frac{\text { Nivel promedio }}{\text { Nivel medio }}>0,66\end{array}$} & $\begin{array}{l}\text { Calificación } \\
\text { de la } \\
\text { iluminación }\end{array}$ \\
\hline $\begin{array}{l}\text { Producción } \\
\text { de dolomita }\end{array}$ & 1000 & 850 & 1000 & 950,00 & 750 & 1,05 & 0,89 & 1,05 & Sí & Adecuada \\
\hline $\begin{array}{l}\text { Molino 6: } \\
\text { Producción } \\
\text { de granito, } \\
\text { marmolina } \\
\text { malla } 400\end{array}$ & 980 & 1050 & 795 & 941,67 & 750 & 1,04 & 1,12 & 0,84 & Sí & Adecuada \\
\hline
\end{tabular}

Elaboración propia 
En la tabla 10 se observa que los resultados arrojados en relación con las medidas de intensidad de iluminación tomados se encuentran por encima del nivel recomendado de lux, ya casi llegando al límite permisible, pero según el criterio de uniformidad la calificación de la iluminación es ADECUADA, es decir, los niveles de iluminación están dentro del rango permitido y además al tratarse de iluminación natural no se considera perjudicial.

\section{Tabla 11}

Evaluación ambiental de iluminación Montajes Técnicos de Colombia

\begin{tabular}{|c|c|c|c|c|c|c|c|c|c|c|}
\hline \multirow{2}{*}{$\begin{array}{l}\text { Puesto de } \\
\text { trabajo }\end{array}$} & \multicolumn{3}{|c|}{$\begin{array}{c}\text { Intensidad } \\
\text { de iluminación } \\
\text { (lux) }\end{array}$} & \multirow{2}{*}{$\begin{array}{c}\text { Nivel } \\
\text { prome- } \\
\text { dio (lux) }\end{array}$} & \multirow{2}{*}{$\begin{array}{c}\begin{array}{c}\text { Nivel reco- } \\
\text { mendado } \\
\text { (lux) }\end{array} \\
750\end{array}$} & \multicolumn{4}{|c|}{$\begin{array}{l}\text { Criterio de uniformidad } \\
\frac{\text { Nivel promedio }}{\text { Nivel medio }}>0,66\end{array}$} & \multirow{2}{*}{$\begin{array}{c}\text { Calificación } \\
\text { de la } \\
\text { iluminación }\end{array}$} \\
\hline & & . & & & & & U, & 0,99 & $c$ & \\
\hline
\end{tabular}

Elaboración propia

En la tabla 11 se observa que los resultados arrojados en relación con las medidas de intensidad de iluminación tomados se encuentran por encima del nivel recomendado de lux, pero según el criterio de uniformidad la calificación de la iluminación es ADECUADA, es decir, los niveles de iluminación están dentro del rango permitido.

Todos los resultados mostrados anteriormente tomados en las diferentes áreas y puestos se encuentran dentro los estándares del Ministerio de la Protección Social y la tabla de calificación diseñada por el Instituto Nacional de Seguridad e Higiene del Trabajo de España, guardando un comportamiento similar, resaltando que una iluminación adecuada es cuando los niveles de iluminación están dentro del rango permitido, el cual es de 500 a 1000 lux.

\section{DISCUSIÓN}

Teniendo en cuenta el desarrollo y los resultados de la investigación, y sobre todo las normas legales que rigen en Colombia, el área de seguridad y salud en el trabajo las cuales fueron acogidas en esta investigación (Resolución 2400, la GTC 8 y el RETILAP), a continuación se presenta la figura 1 con el nivel promedio de lux, en el cual se encuentra el nivel promedio de las mediciones realizadas en cada una de las áreas de trabajo de las ocho empresas para el estudio. También se encuentra demarcado el nivel mínimo, nivel máximo y nivel recomendado. 


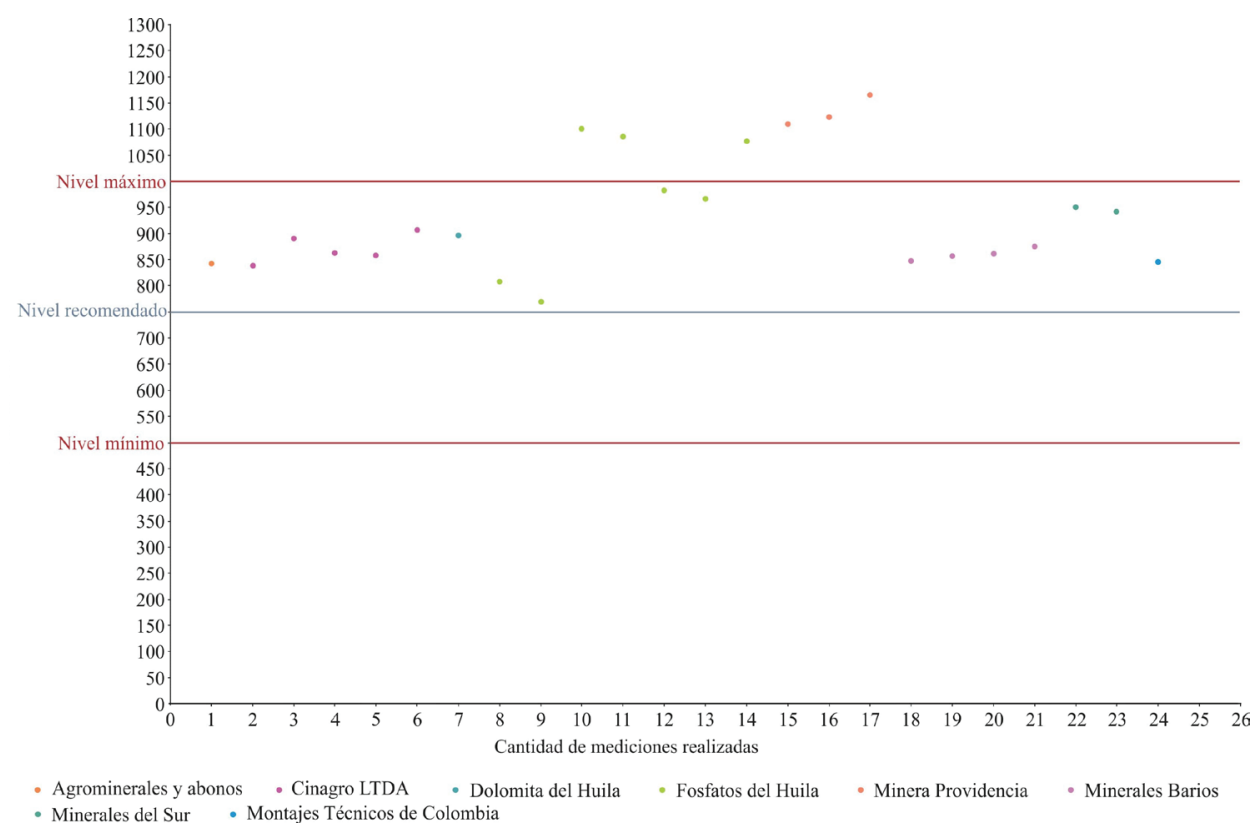

Figura 1. Nivel promedio de lux

Elaboración propia

En la figura 1 se puede observar que en las empresas Agrominerales y Abonos, Cinagro LTDA, Dolomita del Huila, Minerales Barios, Minerales del Sur y Montajes Técnicos de Colombia. El nivel de lux se encuentra dentro de los límites que tiene estipulados la Resolución 2400, lo que clasifica estos puestos de trabajo con una iluminación adecuada para el caso de las empresas Fosfatos del Huila y Minera Providencia. Las mediciones realizadas arrojan resultados por encima del límite máximo (1000 lux); sin embargo, no se considera que la iluminación de estos lugares de trabajo se clasifique como iluminación por encima del intervalo. Como se explica en la GTC 8, si estos niveles son causados por iluminación natural, entonces la iluminación es adecuada; en caso que los niveles de lux sean por iluminación artificial, entonces sí se encontrarían dentro de esta categoría. En este tipo de empresas, es común que la iluminación natural sea protagonista, ya que estas plantas de producción se encuentran ubicadas en terrenos lo suficientemente extensos y con una infraestructura amplia con un diseño que permite el aprovechamiento de la luz solar. La importancia de estar entre los límites permisibles se debe a que la mayor parte de la información que las personas reciben es por medio de la vista, y aunque esta es capaz de adaptarse a unas condiciones deficientes, tener una buena iluminación ayuda a los empleadores a evitar el riesgo para los trabajadores que 
se encuentran expuestos a una mala iluminación y a conseguir un mayor rendimiento en el trabajo y prolongar su salud.

En otros estudios realizados previamente, como Niveles de iluminación en puestos de estudio - Biblioteca sede Candelaria de la Universidad Libre de Colombia, por Beltrán Vera y Rodríguez Borda (2019), que tenía como propósito determinar los niveles de iluminación de estos espacios, a pesar de que se desarrollen en ellos actividades diferentes, se presenta una relación entre las mediciones realizadas y el método, el cual se empleó para llevar a cabo esta investigación. Por lo tanto, en el estudio mencionado se concluye que la uniformidad de la iluminación de la biblioteca no cumple con los estándares que exigen las normas legales y presenta un $73 \%$ de los niveles por fuera de los límites de control -tanto inferiores como superiores-, lo que indica que existe un riesgo para quienes permanecen un tiempo de exposición considerable.

En contraste, los resultados del presente estudio demuestran que las empresas pertenecientes a la industria minera cumplen actualmente los reglamentos técnicos para el funcionamiento, guardando los protocolos de seguridad y salud en el trabajo para proteger la integridad de los trabajadores. En cuanto a la iluminación, los lugares de trabajo se encuentran con iluminación adecuada prevenir accidentes, reducir problemas de salud, mejorar el rendimiento laboral y la concentración.

\section{CONCLUSIONES}

Luego de llevar a cabo la presente investigación, y según la revisión literaria acerca de seguridad y salud en el trabajo, específicamente en lo relacionado a la iluminación en las empresas del sector minero, que tienen por actividad económica la transformación de roca fosfórica en productos de uso agroindustrial (fertilizantes, abonos, etcétera), se puede decir que todas las empresas que participaron en este estudio:

- Cuentan con una iluminación adecuada, lo que garantiza para el trabajador óptimas condiciones de trabajo debido a la excelente iluminación en que se encuentran las áreas y los puestos de trabajo.

- Aprovechan al máximo la luz natural en los talleres de mantenimiento y las áreas de producción.

- La distribución de la iluminación es uniforme y adecuada y cumple con lo recomendado según las leyes y guías técnicas colombianas para cada puesto de trabajo.

Por tanto, las áreas de trabajo que fueron objeto de inspección se clasifican en un nivel aceptable de iluminación. Se llega a dicha conclusión a partir de lo estipulado en la Resolución 2400 y la GTC 8, en donde muestra que los niveles de iluminación adecuados 
para estas áreas deben estar entre los 500 y los 1000 lux, y que el nivel promedio es de 750 lux. Además, si en algún momento la medición da por encima de estos niveles, es por la luz natural que se encuentra en los lugares donde se encuentran ubicadas las plantas de producción.

Finalmente, gracias a los resultados obtenidos mediante las listas de chequeo y mediciones realizadas, analizando la identificación de peligros y evaluación de riesgos, se puede concluir que el riesgo por iluminación deficiente en estas empresas no se presenta, debido a que las actividades derivadas de trabajo son realizadas con la iluminación adecuada, haciendo uso de la luz solar y una correcta la luz artificial, con el fin de preservar un buen ambiente de trabajo. Además de lo dicho anteriormente, la importancia de realizar estudios como estos en la región hace que de cierta manera se genere un control en las empresas, pero con mayor razón en empresas de este sector económico, pues son empresas que de un tiempo prudencial se han convertido en parte importante para el desarrollo económico por ende, realizar seguimiento a diferentes factores de la seguridad y salud en el trabajo hace que cada vez estas condiciones repercutan en una buena salud laboral en las empresas.

\section{REFERENCIAS}

American Industrial Hygienist Association (1959). American Industrial Hygiene Association Journal.

Beltrán Vera, J. F. y Rodríguez Borda, N. E. (2019). Niveles de iluminación en puestos de estudio-Biblioteca Sede Candelaria [tesis de grado, Universidad Libre de Colombia].

Chavarría, R. (2003). lluminación en el puesto de trabajo. Boletín de prevención de riesgos laborales para la Formación Profesional del Instituto de seguridad e higiene. Madrid.

Decreto Reglamentario n. 1072 (2016) Sector del trabajo. Recuperado de https://www. mintrabajo.gov.co/documents/20147/0/DUR+Sector+Trabajo+Actualizado+a+15 +de+abril++de+2016.pdf/a32b1dcf-7a4e-8a37-ac16-c121928719c8

El Huila, una economía que diversifica (30 de julio de 2019). Portafolio. Recuperado de https://www.portafolio.co/economia/el-huila-una-que-se-diversifica-532088

ICONTEC (1994). Guía Técnica Colombiana GTC 8: Electrotecnia. Principios de ergonomía visual. Iluminación para ambientes de trabajo en espacios cerrados. Recuperado de https://www.academia.edu/40868915/GU\%C3\%8DA_T\%C3\%89CNICA_GTC_ COLOMBIANA_8 
ICONTEC (2012). Guía Técnica Colombiana GTC 45: Guía para la identificación de los peligros y la valoración de riesgos en seguridad y salud ocupacional. Recuperado de https:// idrd.gov.co/sitio/idrd/sites/default/files/imagenes/gtc450.pdf

Melgar, A. M., Granados, J. P. y Ruiz, I. A. (2009). Curso de seguridad y salud en el trabajo. Madrid: Editorial Universitaria Ramón Areces.

Ministerio de Minas y Energía de Colombia (30 de marzo, 2010) Reglamento técnico de iluminación y alumbrado púbico RETILAP. Recuperado de https://www.minenergia.gov.co/retilap

Molina Calderón, S. y Ortiz Rojas, H. M. (2019). Análisis de las potencialidades del sector minero del departamento del Huila. Revista Nova, 4, 57-67. https://doi. org/10.23850/25004476.2027

Ortega Alarcón, J. A., Rodríguez López, J. R. y Hernández Palma, H. (2017). Importancia de la seguridad de los trabajadores en el cumplimiento de procesos, procedimientos y funciones. Revista Academia y Derecho, 8 (14), 155-176.

Resolución 2400 (1979). Disposiciones sobre vivienda, higiene y seguridad industrial en los establecimientos de trabajo. Resolución 2400. Recuperado de https:// www.secretariajuridica.gov.co/transparencia/marco-legal/normatividad/ resoluci\%C3\%B3n-2400-1979\#: :text=Descripci\%C3\%B3n\%3A,en\%20los\%20 establecimientos $\% 20$ de $\% 20$ trabajo

Resolución n. 180466 (2 abril del 2007). Modificación del reglamento técnico de instalaciones eléctricas RETIE. Recuperado de http://legal.legis.com.co/document/Inde x?obra=legcol\&document=legcol_759920423021f034e0430a010151f034

The Calibration Centre (2016) Operation Manual Digital lux meter: LX1010B. Recuperado de https://www.thecalibrationcentre.co.uk/wp-content/uploads/2016/02/LX1010B _Luxmeter-Datasheet.pdf 